\title{
Spatial clustering of childhood cancers in Great Britain
}

\author{
E G Knox, E A Gilman
}

\begin{abstract}
Study objectives - Firstly, to identify spatially close pairs and triplets of childhood leukaemias and cancers in Britain. Secondly, to compare pair frequencies with random expectations, identify excesses, and measure the diameters of any clusters. Thirdly, to infer possible causes.

Design and setting - Stratified Poisson analyses of two comprehensive sets of childhood leukaemia and cancer data in Great Britain: seeking home address pairs within particular census enumeration districts (EDs) or postcodes (PCs). Numbers of pairs/triplets of leukaemia registrations sharing single or adjacent EDs were compared with Poisson expectations in national ED strata with different numbers of households (HHs). Pairs/triplets of leukaemia/cancer deaths (and births) sharing a single PC were compared with Poisson expectations in national PC strata with different numbers of postal delivery points (DPs). Same and adjacent house pairs were identified individually among the same PC death pairs. Areal case densities were estimated around a sample of index cases, using their own PC grid coordinates, and those recorded in the central PC directory.
\end{abstract}

Participants - These comprised, firstly, all cases of childhood leukaemia and nonHodgkin lymphoma registered between 1966 and 1983 in England and Wales (ED analysis) and, secondly, all childhood leukaemia and cancer deaths between 1953 and 1980, in England, Wales, and Scotland (PC analyses at birth and at death).

Main results - Short range spatial clustering was demonstrated (a) for leukaemia at place of registration, and (b) for leukaemia and cancer (separately and jointly) at both birth and death addresses. There was evidence of additional case pairing in adjacent PCs. Both data sets showed a relative local pair excess of about $1 \cdot 5$, within diameters of 300 metres. Secondary case densities, measured within 600 metres of a sample of unpaired index cases, were raised by the same ratio. The raised risk then tapered with increasing distance to about $3 \mathrm{~km}$. Forty-four non-twin pairs had died at exactly the same address, far in excess of random expectation. This same house excess was due entirely to 31 sibling pairs. They also showed a relative excess of central nervous system and other solid tumours; but without the exact tumour type sibling concordances sometimes seen in $\mathrm{MZ}$ twins. The sibling pairs were only a small part of the overall excess of same PC pairs.

Conclusions - Short range geographical clustering probably reflects two separate causes of childhood cancer, namely (a) an uncommon familial susceptibility to solid cancers, probably inherited, and (b) a group of long standing focal environmental hazards, most effective within a few hundred metres of the source, but detectable as far as $\mathbf{3} \mathbf{~ k m}$.

\section{(f Epidemiol Community Health 1996;50:313-319)}

A significant excess of pairs and triplets of childhood leukaemias whose registration addresses were separated by very short distances was recently reported. ${ }^{12}$ The numbers observed were about twice the expected value. The 9411 registrations of lymphoid leukaemias and lymphomas in Great Britain between 1966 and 1983 revealed 264 pairs whose postcode (PC) coordinates were less than 150 metres apart. Significant space-time interactions had also been demonstrated in these same data and the diameters of the newly found geographical clusters corresponded with the space-time clusters. ${ }^{3}$

The data had been released by the national registry of childhood tumours (NRCT) for collaborative studies of geographic distributions. ${ }^{4}$ Unfortunately, intractable problems in the data dictated the use of inefficient analytical methods, and left the conclusions in some doubt. The locations themselves were ambiguous because (a) the exact PCs were not released (only their map references), (b) Post Office map coordinates relate only to the "first" address in a PC, and (c) a single map reference is often attached by the Post Office to several PCs. Furthermore, an analysis of map positions alone could not take advantage of the known distribution of the sizes of PCs, and could not accurately separate genuine pairing from that due to heterogeneities of PC sizes. The resulting uncertainties also impinged severely upon subsequent attempts to relate apparent clusters to potential geographically localised environmental hazards. ${ }^{5}$

A preferred approach to detecting clusters would have compared the observed and expected numbers of leukaemia pairs sharing the same PC. The national distribution of numbers of addresses (delivery points (DPs)) per PC has been published ${ }^{6}$ and the expected numbers could be aggregated from stratum specific Poisson expectations. However, this required the 
release of the already recorded PCs, and this was refused by the Department of Health on grounds of confidentiality.

We have therefore adapted this proposed method to census enumeration districts (ED), whose size distribution has also been published $^{7}$; and have re-examined the leukaemia registration data. This is less satisfactory than a PC based analysis because EDs are larger and geographically less specific, so we also sought access to a different data set for which original addresses and PCs were available and applied the originally intended procedures. We report the results of both analyses here.

\section{Methods}

The two data sets used in the study have been described fully elsewhere. ${ }^{4589}$ They consist of:

\section{NATIONAL REGISTRY OF CHILDHOOD TUMOURS (NRCT)}

A file of all 9411 registrations of leukaemias and lymphomas in children aged 0-14 years in England, Wales, and Scotland in the 18 year period 1966-83. The information released for the collaborative studies included the ICD code, the map reference of the "first address" in the registration PC, the date of registration, and the child's sex and age in months: but not the PC itself or the birth date. Unlike the PCs and birth dates, the 1981 census ED codes were not considered a threat to confidentiality, and were also supplied. As in our previous investigations, those registration addresses coded as uncertain or unreliable were excluded, as was an artefactual cluster due to the assembly of armed forces families near a favoured treatment centre; and concordantly affected cotwins. The investigation was also limited to England and Wales because of ED format and size differences in Scotland (ED prefixes 56 and greater) compared with the rest of Great Britain (01 to 55): and because of a similar limitation of the published national distribution (see below). We were left with a working file of $8498 \mathrm{ED}$ specified cases. Unlike our previous studies, all forms of leukaemia and lymphoma were included.

The working file was scanned for pairs and triplets of leukaemia and lymphoma registrations located within the same ED. No contemporary national characterisation of these EDs seems to be available, but the same system was used in 1991 after changes described as minimal. A histogram showing ED size variation in England and Wales is supplied in a User's Guide to the 1991 census. ${ }^{7}$ Numbers of households $(\mathrm{HH})$ ranged from less than 25 to more than 400 . A proportional distribution derived from this histogram and from other basic statistics is shown in table 1 . There were 113196 EDs containing 20.749 million $\mathrm{HH}$, 183.3 per ED. There were 75.07 leukaemias and lymphomas per $1000 \mathrm{ED}$; and 0.4096 per $1000 \mathrm{HH}$. These data provided the basis for a stratified Poisson calculation of the expected numbers of pairs and triplets.
Table 1 Proportional distribution of households in enumeration districts: England and Wales 1991 census

\begin{tabular}{ll}
\hline Households per ED & Proportions of all EDs \\
\hline $0-50$ & 0.008 \\
$51-75$ & 0.021 \\
$76-100$ & 0.034 \\
$101-125$ & 0.046 \\
$126-150$ & 0.082 \\
$151-175$ & $0 \cdot 170$ \\
$176-200$ & $0 \cdot 251$ \\
$201-225$ & 0.233 \\
$226-250$ & $0 \cdot 118$ \\
$251-275$ & 0.025 \\
$276-300$ & 0.008 \\
$301-325$ & 0.003 \\
$326-350$ & 0.001 \\
$351-375$ & 0.001 \\
Over 375 & 0.001 \\
Total & 1.000 \\
\hline
\end{tabular}

Data were extracted from Fig 8.5, p 225, of reference ${ }^{7}$

Poisson calculations (see text) are based upon mid-band values.

\section{OXFORD SURVEY OF CHILDHOOD CANCERS} (osCC)

A file of all 22458 deaths from leukaemias and other cancers in children aged 0-15 years in England, Wales, and Scotland during the 28 year period $1953-80 .^{89}$ This was originally assembled for the Oxford survey of childhood cancers (OSCC). The disadvantageous limitation to fatal cases is ameliorated because three quarters of all cases at this time died within five years ${ }^{10}$ : and regional treatment variations could not yet have caused survivorship clustering. Death certificate data were supplemented through medical records, and often through parental interview. As well as the date and address at death and the ICD code of the tumour and the child's sex, the supplemented records noted the date and place of birth, and the date, age, and place of cancer diagnosis. The proportional distribution of the different cancers is given in table 2 .

PCs and map references at birth and at death were assembled later and each was tagged to indicate the precision of the recorded addresses. Records with uncertain death locations were omitted. Imprecise location of long demolished homes or streets, and the use of "district generic" PCs, could have resulted in false clustering. Duplicate entries, concordantly affected twins, and deaths in Orkney and Shetland, were excluded. This left a working file of 21266 which was scanned for pairs and triplets sharing the same PC either at death or at birth. Where birth addresses were missing or imprecise, they were excluded from the subanalyses of birthplace.

Table 2 Proportions of cases with different diagnoses. Childhood leukaemia and cancer deaths, England, Scotland, and Wales, 1953-80

\begin{tabular}{rll}
\hline 0 & Lymphatic leukaemia & \\
1 & Myeloid leukaemia & 0.23931 \\
2 & Monocytic leukaemia & 0.09123 \\
3 & Other leukaemia & 0.01394 \\
4 & Lymphomas & 0.07426 \\
5 & Wilm's tumour & 0.08736 \\
6 & Central nervous system tumour & 0.05301 \\
7 & Neuroblastoma & 0.16625 \\
8 & Bone tumour & 0.08067 \\
9 & Other solid malignant tumour & 0.03902 \\
10 & Benign/unclassified tumour & 0.10228 \\
\hline
\end{tabular}

Leukaemias and lymphomas (0-4) represent 0.50610 of total. 
Table 3 Numbers* of delivery points (DP) per postcode $(P C)$

\begin{tabular}{llll}
\hline DPs per $P C$ & $P C s$ & DPs per $P C$ & \multicolumn{1}{l}{$P C s$} \\
\hline 1 & 84137 & 16 & 38718 \\
2 & 94544 & 17 & 26710 \\
3 & 64775 & 18 & 33398 \\
4 & 71220 & 19 & 24367 \\
5 & 52207 & 20 & 32529 \\
6 & 61657 & $21-25$ & 110900 \\
7 & 44245 & $26-30$ & 91300 \\
8 & 56458 & $31-35$ & 67000 \\
9 & 40577 & $36-40$ & 52000 \\
10 & 48051 & $41-45$ & 35500 \\
11 & 35533 & $46-50$ & 24500 \\
12 & 50396 & $51-55$ & 15700 \\
13 & 31891 & $56-60$ & 14500 \\
14 & 38656 & $61-65$ & 10400 \\
15 & 30353 & $>65$ & 15532 \\
Total PCs & 1397754 & & \\
Total DPs 23845162 & & \\
Mean DP per PC $=17.06$ & & \\
\hline
\end{tabular}

* Numbers of PCs with DPs up to 20 are exact and were taken from reference ${ }^{6}$, table 2.3. For DP>20, the estimates were taken from a graphic presentation (Fig 2.3, reference ${ }^{6}$ ) and adjusted to reconcile them with the overall totals.

Poisson estimates of expected pairs (see text) are based upon estimated single level values rather than the five level DP bands in the above table.

An adaptation of a published national distribution of delivery points (DPs) per residential PC, is shown in table $3 .^{6}$ Central PC directories are revised continually and the total did not correspond exactly with our own version, so we applied the published proportional distribution to our own total. The 21266 deaths were distributed across 1372359 residential PCs in England, Wales, and Scotland: 15.50 per 1000 PCs; and across 23845162 DPs giving 17.06 DPs per PC and 0.8918 deaths per 1000 DPs.

\section{DATA OVERLAP}

The two data sets overlapped, but the cooperative use of common serial numbers during their assembly identified the 2049 duplicated cases. They amounted to $24 \cdot 1 \%$ of the NRCT and $9.63 \%$ of the OSCC working files. Studies of mutual proximities require examination of all possible pairs of cases and, in this numerically inflated context, the degree of overlap was even less. It amounted to $5 \cdot 8 \%$ of all possible pairs in the first set and $0.9 \%$ of all those in the

Table 4 Distribution of electoral district (ED) separations of leukaemia/lymphoma pairs. Childhood leukaemiallymphoma registrations: England and Wales 1966-83

\begin{tabular}{|c|c|c|c|c|}
\hline ED suffix difference (d) & Observed pairs & Expected pairs* & $O / E$ ratios & $\begin{array}{l}\text { Mean inter-pair } \\
\text { distances }(\mathrm{km}) \dagger\end{array}$ \\
\hline $\begin{array}{c}\quad 0 \text { (same ED) } \\
1 \\
2 \\
3 \ldots 5 \\
6 \ldots 10 \\
11 \ldots 15 \\
16 \ldots 20 \\
21 \ldots 30 \\
31 \ldots 40 \\
41 \ldots 98\end{array}$ & $\begin{array}{r}520 \\
641 \\
608 \\
1483 \\
1546 \\
848 \\
475 \\
381 \\
93 \\
54\end{array}$ & $\begin{array}{r}338 \cdot 8 \\
643 \cdot 2 \\
591 \cdot 8 \\
1453 \cdot 6 \\
1555 \cdot 7 \\
897 \cdot 8 \\
539 \cdot 7 \\
461 \cdot 0 \\
113 \cdot 5 \\
54 \cdot 0\end{array}$ & $\begin{array}{l}1.535 \\
0.997 \\
1.027 \\
1.020 \\
0.994 \\
0.945 \\
0.880 \\
0.826 \\
0.820 \\
1.000\end{array}$ & $\begin{array}{l}0 \cdot 195 \\
0 \cdot 685 \\
0 \cdot 790 \\
0 \cdot 890 \\
1 \cdot 114 \\
1 \cdot 326 \\
1 \cdot 710 \\
1 \cdot 752 \\
2 \cdot 149 \\
2 \cdot 439\end{array}$ \\
\hline Total & 6649 & $6649 \cdot 0$ & - & $1 \cdot 053$ \\
\hline
\end{tabular}

* The expected d distribution is based on the distribution of suffix differences among all possible case pairs in the entire $(\mathrm{E} \& \mathrm{~W})$ file, irrespective of whether the non-suffix parts of their ED codes matched. Expected numbers with different " $d$ " were calculated from the primary distribution of numbers of different suffixes within the file rather than directly counted (see Appendix). However, two directly counted alternative estimations of expected values were also made, and they gave similar results. The first was based upon all pairs with matching region codes (first 2 digits) but non-matching area codes (next 4 alpha characters). The second used pairs which matched both on prefix and on the first pair of alpha characters, but not on the second pair. The first gave a $\mathrm{d}=0 \mathrm{O} / \mathrm{E}$ ratio of 1.621 , and the second gave 1.696

+ Calculated from PC derived map references of the pairs. second. The geographical definitions of a "close" pair differ in the two sets, as do the tumour types. For analytical purposes, the two studies can be regarded as independent.

\section{Results}

ED SHARING AMONG LEUKAEMIA/LYMPHOMA REGISTRATIONS

Among the 8498 cases accepted for analysis, clusters of two or more registrations occurred in 448 different EDs: 415 had two, 31 had three, and 2 had four $(415 \times 1+31 \times 3+$ $2 \times 6=520$ separate case pairs: involving $415 \times 2+31 \times 3+2 \times 4=931$ children). If the risk were equal in all $113196 \mathrm{EDs}$, with 75.07 cases per $1000 \mathrm{ED}$, then the Poisson expectations would be $303.46 \mathrm{ED}$ clusters of two or more $(\mathrm{O} / \mathrm{E}$ ratio $=1.48: \mathrm{p}<0.0001)$, including 7.55 clusters of three or more $(\mathrm{O} / \mathrm{E}$ ratio $=4 \cdot 37: p<0 \cdot 0001$ ).

Corrected estimates of expected numbers were obtained by aggregating Poisson calculations within each layer of the $\mathrm{HH} / \mathrm{ED}$ distribution shown in table 1 . Stratum specific Poisson parameters were calculated as $\mathrm{HH} \times 0.4096 / 1000$ (see above). The new expected values were 321.6 clusters of two or more $(\mathrm{O} / \mathrm{E}$ ratio $=1.39: \mathrm{p}<0.0001)$, including 8.89 of three or more $(\mathrm{O} / \mathrm{E}$ ratio $=3 \cdot 71$ : $\mathrm{p}<0.0001)$. The $\mathrm{O} / \mathrm{E}$ narrowing achieved by excluding this major heterogeneity is quite modest. It is unlikely that additional population heterogeneities within individual $\mathrm{HH}$ strata could explain the whole of the remaining excess.

ED codes comprise an initial, 2 digit, region prefix followed by a 4 letter area code and a 2 digit district suffix. As well as same ED pairs, we identified pairs which spanned suffix adjacent EDs, whose codes were otherwise identical, and also pairs with successively greater suffix differences. We later confirmed that increasing suffix differences were related to linear distance apart. If ED pairs were random coincidences within wider zones of raised incidence, then we should see a gradual decrease of the excess pairs as distances and suffix differences widened; but if they are related to hazards of very short effective radius, then the excesses should be limited to the shortest intervals.

The results of this enquiry, given in tables 4 and 5 , compare the observed distribution of suffix differences with an expected distribution. The latter is based upon the suffix combinations of all registration pairs, irrespective of whether they matched in other respects (see table 4 and the Appendix). Alternative methods of calculation, noted in the footnote to table 4, gave the same results. This comparison confirms the results of the earlier Poisson based demonstrations of ED sharing. Using the associated PC coordinates of the case pairs it also shows a clear relationship between the widening suffix differences of case pairs and their linear distances apart. The relative excess is sharply concentrated among the same ED pairs at distances centred on 200 metres, although relative frequencies descend further towards the tabulation limits of about $2 \mathrm{~km}$. Table 5 shows 
Table 5 Electoral district suffix differences (0..5) and linear distances. Childhood leukaemia/lymphoma registrations: England and Wales 1966-83

\begin{tabular}{|c|c|c|c|c|c|c|}
\hline \multirow[t]{2}{*}{$\begin{array}{l}\text { Inter-pair } \\
\text { distance }(\mathrm{km})\end{array}$} & \multicolumn{6}{|c|}{$\begin{array}{l}\text { Leukaemiallymphoma pairs } \\
\text { Suffix difference }\end{array}$} \\
\hline & 0 & 1 & 2 & 3 & 4 & 5 \\
\hline $\begin{array}{r}0.0-0.05 \\
-0.10 \\
-0.20 \\
-0.30 \\
-0.40 \\
-0.50 \\
-0.60 \\
-0.70 \\
-0.80 \\
-0.90 \\
-1.00 \\
-1.50 \\
-2.00 \\
-5.00 \\
>5.00\end{array}$ & $\begin{array}{r}140 \\
79 \\
128 \\
92 \\
36 \\
17 \\
7 \\
5 \\
1 \\
1 \\
2 \\
4 \\
5 \\
3 \\
0\end{array}$ & $\begin{array}{r}3 \\
11 \\
65 \\
125 \\
112 \\
76 \\
56 \\
37 \\
38 \\
19 \\
12 \\
36 \\
19 \\
21 \\
11\end{array}$ & $\begin{array}{r}0 \\
6 \\
37 \\
66 \\
65 \\
86 \\
66 \\
65 \\
52 \\
31 \\
22 \\
49 \\
23 \\
35 \\
5\end{array}$ & $\begin{array}{r}0 \\
6 \\
21 \\
46 \\
66 \\
61 \\
60 \\
39 \\
51 \\
36 \\
41 \\
73 \\
20 \\
17 \\
4\end{array}$ & $\begin{array}{r}0 \\
6 \\
12 \\
42 \\
41 \\
65 \\
49 \\
42 \\
46 \\
28 \\
29 \\
77 \\
35 \\
43 \\
5\end{array}$ & $\begin{array}{r}0 \\
1 \\
12 \\
28 \\
29 \\
46 \\
39 \\
44 \\
41 \\
34 \\
31 \\
64 \\
22 \\
29 \\
2\end{array}$ \\
\hline Totals & 520 & 641 & 608 & 541 & 520 & 422 \\
\hline
\end{tabular}

that almost all of the same ED pairs, and therefore of the excess pairs, are within mutual distances of 400 metres.

\section{LEUKAEMIA AND CANCER DEATHS}

$P C$ sharing

The operational file of 21266 deaths from leukaemia or cancer was searched for all instances where two or more cases of any type shared the same PC. There were 476 twos and nine PC-sharing threes: 485 sets of two or more $(476 \times 1+9 \times 3=503$ separate pairs: involving $476 \times 2+9 \times 3=979$ separate children).

A homogeneous Poisson series based on the overall rate of 15.50 cases per 1000 PCs gives an expected pair + triplet rate of 118.82 per million PCs, amounting nationally to 163.07 same PC sets of two or more. The observations exceed the expectation by a factor of 2.97 $(\mathrm{p}<0.0001)$.

A correction for PC heterogeneity was based upon aggregated Poisson expectations calculated separately for each DP level using Poisson parameters of $\mathrm{DP} \times 0 \cdot 8918 / 1000$ (see above). The expected number of pairs or greater was now $283 \cdot 88$, and the $\mathrm{O} / \mathrm{E}$ ratio $1 \cdot 71$ $(\mathrm{p}<0.001)$. The expected number of triplets or greater was 3.46 , giving a ratio of $2 \cdot 60$ $(\mathrm{p}<0.001)$.

The tumour type combinations of the 503 separate same PC pairs are shown in the first panel of table 6. PC sharing was investigated further using address at birth, where this was adequately recorded, and these results are given in the lower panels. The expected proportional distributions were based on the type distribution shown in table 2 . The minor discrepancies are not statistically significant, and for each address combination the excess was spread almost equally across the reticulo-endothelial pairs, the solid tumour pairs, and the cross pairs. A detailed tabulation of all 11 individual tumour types and all 66 cross pairs likewise showed no relative clustering preferences, positive or negative.

Many children were born and died at the same address, resulting in confounding between death pairing, birth pairing, and cross pairing. Their statistical separation depends
Table 6 Tumour type combinations in same postcode pairs. Childhood leukaemia and cancer deaths: England, Scotland, and Wales, 1953-80

\begin{tabular}{|c|c|c|c|c|}
\hline Pair types* & $L L$ & $L S$ & $S S$ & Total \\
\hline $\begin{array}{l}\text { Death-death } \\
\text { Observed } \\
\text { Expected } \dagger\end{array}$ & $\begin{array}{l}\text { esses } \\
135 \\
128.8\end{array}$ & $\begin{array}{l}256 \\
251 \cdot 5\end{array}$ & $\begin{array}{l}112 \\
122 \cdot 7\end{array}$ & 503 \\
\hline $\begin{array}{l}\text { Birth-birth a } \\
\text { Observed } \\
\text { Expected } \dagger\end{array}$ & $\begin{array}{l}\text { ses } \\
\quad 64 \\
64 \cdot 8\end{array}$ & $\begin{array}{l}113 \\
126 \cdot 5\end{array}$ & $\begin{array}{l}76 \\
61 \cdot 7\end{array}$ & 253 \\
\hline $\begin{array}{l}\text { Birth-death } \\
\text { Observed } \\
\text { Expected } †\end{array}$ & $\begin{array}{l}\text { sses } \\
154 \\
147 \cdot 5\end{array}$ & $\begin{array}{l}275 \\
288 \cdot 0\end{array}$ & $\begin{array}{l}147 \\
140 \cdot 5\end{array}$ & 576 \\
\hline
\end{tabular}

* LL pairs are those in which both children had either leukaemia or lymphoma. In LS pairs, one had leukaemia or lymphoma and the other had a solid tumour. SS signifies two solid tumours. t Expected values are proportional binomial distributions of ascertained totals, using tumour proportions given in table 2 . Pairs of children with identical death and birth $\mathrm{PC}$ are included both in panel 1 and in panel 2 .

Table 7 Rates* of postcode pairing at birth and death addresses. Childhood leukaemia and cancer deaths. England, Scotland, and Wales, 1953-80

Change-status Address pair types

\begin{tabular}{|c|c|c|c|c|c|}
\hline \multirow{2}{*}{ of pairt } & \multicolumn{5}{|l|}{$x_{0}$} \\
\hline & Birth-birth & & Birth-death & & Death-death \\
\hline $\begin{array}{l}N \text { N } \\
N \text { C } \\
\text { C C }\end{array}$ & $\begin{array}{l}2 \cdot 82 \\
2 \cdot 27 \\
2 \cdot 82\end{array}$ & $=$ & $\begin{array}{l}2.82 \\
1.96 \\
1.96\end{array}$ & $=$ & $\begin{array}{l}2 \cdot 82 \\
1 \cdot 79 \\
2 \cdot 09\end{array}$ \\
\hline Other $\neq$ & & - & & 0 & \\
\hline
\end{tabular}

possible pairs.

+ Rates are measured in three classes of pair NN, NC, and CC Rates are measured in three classes of pair NN, NC, $¥$ Those in which one or both of the pair had an inexact or $¥$ missing record of the birth address.

chiefly upon an analysis of those who moved Only 9176 had changed PCs, while 9193 had not, and the remainder were indeterminate. A complex analysis of these three groups, separating the death-death, birth-birth and birthdeath PC sharing pairs, is summarised in table 7. The cluster frequencies used for comparing the different groups are the ratios between same PC case pairs and all identified case pairs of the relevant type. This is the natural denominator for comparing coterminous data sets with different geographical densities, and it avoids an obvious artefact. For example, a doubling of the numbers of cases available for a pair count (for example, through extending the period of the investigation) will quadruple the numbers both of available pairs and of the close pairs to be found among them. A simple ratio of close pairs (quadrupled) to total cases (doubled) would show a false apparent doubling of the close pair rate. The use of all pairs as the denominator avoids this problem.

The highest rates occurred when at least one of the pairs lived at one address throughout, and especially when both did so $(2.82$ per million pairs). Among the movers, the rates were greater among birth-birth PC pairs than among the birth-death and death-death pairs. However, all the rates were excessive when set against our earlier calculations. The analogous denominator for the 503 death pairs in the total group is $226 \cdot 1$ million pairs ( $21266 \times$ $21255 / 2$ ) and the corresponding rate is $2 \cdot 22$ per million pairs. For the 283.88 expected cases, set on the same denominator, the 
corresponding rate is 1.26 per million pairs. This last value (with $S E M=0.075$ ) provides a comparative random standard for table 7 . The excesses probably reflect persistent or intermittent local hazards whose effectiveness depends in part upon the duration of residence. The effect is possibly greater in the early years of life, or during gestation, but seems to operate at all ages.

\section{Cancer houses}

All pairs with identical death PCs were listed and the original records were searched for the exact addresses and names. We hoped to describe the distances between pairs within individual PCs and to settle the question of very localised risks restricted to a few tens of metres, including those associated with particular "cancer houses". It was also necessary to identify and exclude clusters in large child-resident institutions such as boarding schools, or homes for the handicapped. The latter could be especially important where they accept children with Down syndrome because of the 20 fold risk of leukaemia associated with this condition. In the event, no institutional clusters were found. We also needed to identify affected sibs.

Excluding twins, there were 44 confirmed pairs with the same death address during the 28 years, contrasting with a Poisson expected $9.48(\mathrm{O} / \mathrm{E}=4.64: \mathrm{p}<0.001)$; based on the overall rate of 0.8918 per 1000 DPs. The calculated expectation is in fact an over estimate since the occurrence of one case reduces the number of children subsequently at risk.

In 464 of the 503 independent, same PC pairs, both children lived in numbered houses in the same named street. House number differences $(0,1,2,3,4 \ldots)$ were counted. The frequencies of the successive number differences were $44,14,25,13,27,13,21,10$, $17,12,19,10,20,8,13,7,12$. . . etc. The expected national proportions of houses separated by $0,1,2,3,4,5, \ldots \mathrm{n}$ other houses were calculated from the PC size distribution shown in table 3 , as for houses arranged in a straight number sequence. Successive expected frequencies were $15 \cdot 8,29 \cdot 9,28 \cdot 1,26 \cdot 5,24 \cdot 9$, $23 \cdot 5,22 \cdot 1,20 \cdot 8,19 \cdot 6$. . . etc. Real streets are usually numbered in steps of 2 within odd and even series on opposite sides, so exact comparison is not possible. However, the "cancer houses" excess shown by the Poisson analysis is confirmed (for " $\mathrm{d}$ " $=0: \mathrm{O} / \mathrm{E}=44 / 15 \cdot 8$ ); while same side neighbours (separations $=2,4,6$, etc) followed a linear gradient sufficiently close to that calculated. Odd numbered separations are probably a mix of same side and opposite side near neighbours. The even-d series is derived from odd-odd $(\mathrm{OO}=174)$ and even-even $(E E=142)$ house number pairs; it substantially exceeded the odd-d series composed of oddeven $(\mathrm{OE}=148)$ pairs. This probably reflects a preference for allocating one sided PCs, rather than a hazard restricted to one side of the street, and it indicates an unidentified number of $\mathrm{OE}$ close pairs bridging boundaries between adjacent odd and even PCs.
Comparison of the observed and expected house number separations shows that the hazard determining the "cancer house" pairs is extremely localised. The effect does not extend preferentially to the house next door or across the street. Of the 44 same address pairs, 31 were identified as non-twin sib pairs while 13 were from unrelated families. The non-sib pairs are no more frequent than "expected" but the sib pairs exhibit a sharp excess. The same house excess is thus associated specifically with concordant sibships. It is difficult to show formally whether this is because a house associated hazard must frequently strike sibs; or because the effects of a family associated risk must frequently occur at the same address, but there are two items of evidence in favour of the latter.

Firstly, for 16 of the 31 sib pairs, the first affected child was already dead when the second affected child was born. There was no possibility in any of these cases of a common exposure to a transient lethal hazard; and, given the large numbers of children who moved house, a longer lasting house based hazard should have resulted in a noticeable transfer of risk between successive families occupying the same dwelling.

Secondly, the distribution of cancer types among the sib pairs differed from that in table 2. Among the 62 sibs ( 31 pairs) there were 49 solid tumours (groups 5-10) against an expected $30.6(p<0.01)$. Among these solid tumours, group 6 (CNS) dominated, with 25 cases against an expected 10.3 ( $<<0.001)$. These $31 \mathrm{sib}$ pairs included six brain/brain pairs and nine brain/other solid tumour pairs, of which five were sarcomas of bone or muscle. The 26 non-sib cases (in 13 same house pairs) included 15 solid tumours ( 12.8 expected) of which six were CNS tumours ( 4.3 expected). The predominance of solid tumours, and especially brain tumours, among affected sib pairs has been reported previously within this same set of data in a comprehensive study of both dead and surviving children, irrespective of their locations. ${ }^{11}$

The question arises whether the family associated, same house, solid tumour excess is genetically or environmentally determined. Eligible environmental hazards include vertical virus transmission from the mother, or fetal injury from an abnormal maternal metabolite, or a drug prescribed for a long standing condition (for example, epilepsy). None of the fatal pairs had had retinoblastoma - a known genetic risk - but in four pairs both children had (or probably had) a reticulosis or malignant histiocytosis. It was described specifically in two pairs as "familial" in type. Apart from these four pairs there was no clear tendency to share exactly the same tumour, the usual circumstance when $\mathrm{MZ}$ twins are concordantly affected. Only one of the brain-brain pairs was described in a manner to suggest they might be type concordant - two medulloblastomas; and there were two pairs of concordant acute leukaemias, neither of them type concordant. If there is a genetic element then, apart from the histiocytoses, it is not simply deterministic. 
Table 8 Relative case densities at successive radial distances from index cases. Childhood leukaemia and cancer deaths: England, Scotland, and Wales, 1953-80

\begin{tabular}{lccccccc}
\hline \multicolumn{7}{c}{$K m$ distant } \\
\hline Reference points (n) & -0.3 & -0.6 & -1.0 & -2.0 & -3.0 & -5.0 & -10.0 \\
Random cases (700) & 1.24 & 1.24 & 1.18 & 1.17 & 1.07 & 1.01 & 1.00 \\
Case pairs + triplets (503) & 1.50 & 1.40 & 1.29 & 1.25 & 1.11 & 1.05 & 1.02 \\
\hline
\end{tabular}

Relative case densities are the ratios between the secondary case densities per 1000 postcodes, around the index points, and the similar densities around a random set of 500 PCs extracted
from the central PC directory. Reference cases, reference pairs, and reference triplets are excluded from the density calculations.

\section{Radial risk diffusion}

Even when sib pairs are disregarded, PC sharing is still excessive. The numbers of same PC and same ED pairs, the evidence from house numbers of additional pairs crossing PC boundaries, contrasting with the apparent paucity of ED groups with diameters greater than 300-400 metres, suggest the existence of hazards with effective radii up to about 200 metres; but not much more. However, there was also some evidence in tables 4 and 5 of tapering risks up to about $2 \mathrm{~km}$, and these methods clearly do not critically address the question of co-existing hazards whose effects range beyond the limits of these tabulations.

The question of larger radius groupings was therefore approached as follows. The map references of a random sample of 700 leukaemias and cancers were selected as reference points and all other cases and all other PCs were then identified in successive closely spaced annuli around them. After excluding the reference cases themselves, secondary densities of cases per $1000 \mathrm{PCs}$ were calculated at different radial distances up to $10 \mathrm{~km}$. A sample of $500 \mathrm{PCs}$ was then randomly selected from the central PC directory and used as a control set of reference points. Radial densities were measured in the same way, except that there were no index cases to omit. Risk levels at successive distances surrounding the deaths were expressed as ratios between the case-PC based and the random-PC based densities (table 8).

These ratios show that the narrow concentrations of leukaemia and cancer deaths demonstrated above are probably embedded in wider risk zones. The short range excesses corresponded with those already demonstrated, reducing to a half in about $2 \mathrm{~km}$, but remaining detectable up to about $3 \mathrm{~km}$. In none of these examinations did the use of different tumour types as reference points, or as the basis for measuring the radial gradients, make any difference to the results. The process was repeated for reference points corresponding with pair sharing and triplet sharing PCs, and the results were similar, or perhaps a little more widely spread.

This form of analysis does not distinguish clearly between true risk level variations and self selection effects arising from local concentrations of large PCs. The results should not be construed as independent confirmation of clustering (if such were needed) but as an indication of the upper effective ranges - about $3 \mathrm{~km}$ - of some of the cluster determining hazards. The correspondence of the short range $\mathrm{O} / \mathrm{E}$ ratios with those found with heterogeneity correcting methods indicates that demographic artefacts incurred by using random PCs as a comparison group are less severe than might have been feared.

\section{Discussion}

Our previous search for spatial clustering in childhood leukaemia was compromised by limited geographic specifications in the available data and inadequate control of demographic heterogeneities. Clusters of cases might have reflected local concentrations of children at risk rather than high levels of risk. The objective of the present enquiry was to set the issue on a more secure base.

Spatial clustering of leukaemias and lymphomas (L) was confirmed in two independent data sets using two different population groupings, namely census EDs and PCs. The geographic scales of the clusters were similar, and similar again to the space-time clusters demonstrated on another occasion. The second data set showed that the clustering also involved the solid cancers $(C)$, and there were same PC excesses of L-L pairs, of C-C pairs, and of cross pairs $(L-C)$ involving one of each. These combined excesses were found in death address pairs, in birth address pairs, and in address cross pairs involving one of each. The risks were related to duration of residence, and the results in children who moved house suggested that exposures close to the birth address were marginally more dangerous than those close to the death address.

The indistinguishable spatial patterns of the leukaemias and the solid cancers imply the existence of a hazard (or hazards) affecting both groups equally; and therefore of a readily diffusable hazard with access to many tissues. This corresponds with the findings in fetal medical irradiation, the only large scale determinant so far identified, ${ }^{9}$ and one which likewise has unrestricted tissue access. Unlike fetal irradiation, however, the new hazard is effective in later as well as in early childhood and is not specific to prenatal exposure. Spacetime interactions were likewise evident at early and late ages ${ }^{12}$ and probably share common origins with the purely geographical clusters. Space-time clustering was limited to the leukaemias, but this difference from the solid cancers may be less than fundamental. It perhaps reflects a relatively short and constant response time, following exposure to an intermittent hazard; compared with a longer and more variable interval for the other cancers.

There is still a residual, if much reduced, problem of demographic heterogeneity. Cumulative child years at risk must vary within the stratum specific PCs and EDs studied here, but such data are not available. In previous searches for extra-Poissonian variation in the NRCT data, a requirement by the investigators for known population denominators enforced the use of much larger groups. They used electoral wards ${ }^{13}$ or large aggregations of EDs. ${ }^{14}$ The evidence for clustering at these coarse levels was weak or ambivalent, as indeed might be expected from our own results. A major reassurance against demographic artefact in 
our own fine scale analysis, with no access to accurate denominators, is the consistency of results obtained from different data sources, different population groupings, and complementary modes of analysis.

The causes of the same-house, sib-pairs probably differ from those responsible for other PC and ED pairs. Their occurrence in sibs whose lives did not overlap, the unusual tumour types, and their extreme localisation to a single house with no local diffusion, indicate an intrafamilial source rather than a hazard of the premises. However, frequent tumour type nonconcordance does not suggest a directly deterministic genetic defect, but perhaps repeated fetal exposure to a long term maternal hazard such as a chronic virus infection or a long term fetal toxic drug prescription, or the pathological metabolite of an abnormal maternal genotype; or else a transmitted genetic defect operating indirectly through the immune system or the cell division process. Such indirect genetic mechanisms have been suggested by others, and for the same reasons. ${ }^{11}$

The general clustering pattern among case pairs sharing the same ED or PC had a dominant component which spanned diameters of about 200-300 metres. This, however, is probably superimposed upon wider zones of elevated risk with radii up to about $3 \mathrm{~km}$. When the sib pairs were excluded, there was no evidence of additional very short range ambient risks covering adjacent or nearly adjacent houses, and limited to a few tens of metres.

In a previous study, ${ }^{5}$ the environments of clusters were examined for apparent hazards in order to seek confirmation that the clusters were real. This introduced a circularity which then compromised comment on the nature of the hazards themselves. The major importance of the present findings is that we now know that the clusters exist, and that there must be at least one class of local environmental hazard, so that the objectives of future environmental examinations can be limited solely to the identification of the sources. There are many possible candidates, including some already suggested. ${ }^{5}$ The radial case density method and the new data set employed here offer a more secure basis for investigating these questions than that previously available.

We thank Dr G J Draper and the Childhood Cancer Research Group for providing the data on which the ED analyses were based and Dr A M Stewart for access to the data of the Oxford survey of childhood cancers. The postcoding of the OSCC records was part of a research programme supported by the Medical Research Council and by the Three Mile Island Public Health Fund (USA). EGK's expenses during the current work were defrayed through a Leverhulme Emeritus Research Fellowship.

\section{Appendix}

Derivation of expected numbers of electoral district (ED) suffix differences as used in table 4.
Observed numbers (n) of EDs with different suffixes (s) in the total leukaemia/lymphoma registration set are represented as $n_{1}, n_{2}, n_{3}$. $\ldots \mathrm{n}_{\mathrm{s}} \ldots \mathrm{n}_{\mathrm{s}}$. From this we can calculate numbers of all possible pairs of cases with same ED suffix, irrespective of matching/non-matching of the prefix and area codes of the ED. At each $s$ level this is $n(n-1) / 2$ and the overall aggregate is

$$
\sum_{s=2}^{s} n_{s}\left(n_{s}-1\right) / 2
$$

Numbers of pairs with suffixes separated by "d" are obtained from $\mathrm{n}_{\mathrm{s}}$ cross products. Those separated by $\mathrm{d}=1$ are

$$
\sum_{s=1}^{s-1} n_{s} n_{s+1}
$$

For each larger $\mathrm{d} \ldots$

$$
\sum_{s=1}^{s-d} n_{s} n_{s+d}
$$

1 Knox EG, Gilman EA. Leukaemia clusters in Great Britain. (2) Geographical concentrations. F Epidemiol Community Health 1992;46:573-6.

2 Gilman EA, Knox EG. Temporal-spatial distribution of childhood leukaemias and non-Hodgkin lymphomas in Great Britain. In: The geographical epidemiology of childhood leukaemia and non-Hodgkin lymphomas in Great Britain, leukaemia and non-Hodgkin lymphomas in Great Britain, 1966-83. Studies on medical
53. London: HMSO, 1991 .

3 Knox EG, Gilman EA. Leukaemia clusters in Great Britain. (1) Space-time interactions. F Epidemiol Community Health 1992;46:566-72.

4 Draper GJ ed. The geographical epidemiology of childhood leukaemia and non-Hodgkin lymphomas in Great Britain, 1966-83. Studies on medical and population subjects no 53. London: HMSO, 1991

5 Knox EG. Leukaemia clusters in childhood: geographical analysis in Britain. $\mathcal{F}$ Epidemiol Community Health 1994; 48:369-76.

6 Raper JF, Rhind DW, Shepherd JW. Postcodes: the new geography. Harlow and London: Longman, 1992.

7 Cole $\mathrm{K}$. The local area base and small area statistics, pp 201-47. In: Dale A, Marsh C eds. The 1991 census pp 201-47. In: Dale A, Marsh C

8 Stewart AM, Webb J, Hewitt D. A survey of childhood malignancies. BMF 1958;i:1495-508.

malignancies. BMf 1958;i:1495-508.
Knox EG, Stewart AM, Kneale GW, Gilman EA. Prenatal irradiation and childhood cancer. $\mathcal{F}$ Radiol Prot 1987;7: 177-89.

10 Stiller CA. Population based survival rates for childhood cancer in Britain. BMF 1995;309:1612-16.

11 Draper GJ, Heaf MM, Kinnier-Wilson LM. Occurrence of childhood cancers among sibs and estimation of familial risks. F Med Genet 1977;14:81-90.

12 Gilman EA, Knox EG. Childhood cancers: space-time distribution in Britain. F Epidemiol Community Health 1995; 48:158-63.

13 Alexander FE. Investigations of localised spatial clustering, and extra-Poissonian variation. In: Draper GJ ed. The geographical epidemiology of childhood leukaemia and nongeographical epidemiology of childhood leukaemia and nonHodgkin lymphomas in Great Britain, 1966-83. Studies on medical

14 Black RJ, Sharp L, Urquhart JD. An analysis of the geographical distribution of childhood leukaemia and nonHodgkin lymphoma in Great Britain using areas of approximately equal population size. In: Draper GJ ed. The geographical epidemiology of childhood leukaemia and nonHodgkin lymphomas in Great Britain, 1966-83. Studies on medical and population subjects no 53. London: HMSO, 